\title{
Constraints on ultrahigh-energy cosmic ray mass composition using cosmogenic neutrinos
}

\section{Saikat Das}

Astronomy \& Astrophysics Group, Raman Research Institute, Bengaluru 560080, India

E-mail: saikatdaserri.res.in

\section{Soebur Razzaque*}

Centre for Astro-Particle Physics (CAPP) and Department of Physics, University of

Johannesburg, P.O. Box 524, Auckland Park 2006, South Africa

E-mail: srazzaque@uj.ac.za

\section{Nayantara Gupta}

Astronomy \& Astrophysics Group, Raman Research Institute, Bengaluru 560080, India

E-mail: nayan@rri.res.in

Ultrahigh-energy cosmic rays (UHECRs), with energy $\gtrsim 10^{18} \mathrm{eV}$, propagate over cosmological distances, rendering them susceptible to interactions with the cosmic photon backgrounds that produce secondary particles, viz., neutrinos and gamma-rays. The sources, as well as the mass composition of UHECRs, can be constrained by probing these cosmogenic fluxes that extend to energies exceeding $\sim 10^{18} \mathrm{eV}$. The neutrinos, being weakly interacting, travel unhindered and undeflected by extragalactic or Galactic magnetic fields. We fit the observed UHECR spectrum as measured by the Pierre Auger Observatory (PAO) by simulating the propagation of UHECRs of various mass compositions at injection, from different source distributions. We also calculate the cosmogenic neutrino spectrum for the same UHECR parameters, fitting the PAO data. The neutrino spectrum varies depending on the UHECR mass composition and source properties. Although the currently operating detectors do not reach the necessary sensitivity for observing cosmogenic neutrinos in all cases, a few parameter sets producing relatively high fluxes are already constrained by the flux upper limit imposed by 9 -yrs of IceCube data. We also explore possibilities to constrain the UHECR abundance fraction of light nuclei at injection by identifying the fluxes of individual neutrino flavors in future detectors. Although the contribution of neutron beta decay to the cosmogenic neutrino spectrum is insignificant, it shifts the ratio of the fluxes of individual flavors obtained at earth from their constant values; thus serving as a discriminator between different mass composition models. The interactions leading to the production of neutrinos also leave their imprint on the observed UHECR spectrum. This can further explain the origin of cutoff in the UHECR spectrum at the highest energies.

36th International Cosmic Ray Conference -ICRC2019-

July 24th - August 1st, 2019

Madison, WI, U.S.A.

\footnotetext{
* Speaker.
} 


\section{Introduction}

Several candidate source classes have been proposed that are capable of accelerating particles

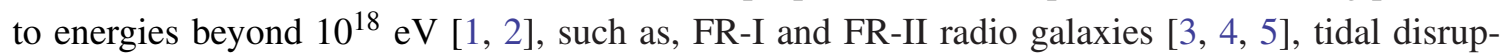
tion events [6,7], young neutron stars [8, 9, 10], gamma ray bursts [11, 12, 13, 3], low-luminosity GRBs [13, 14], hypernovae [15, 16], active galactic nuclei [17, 3, 18], etc. For energies above 39 $\mathrm{EeV}$, the Pierre Auger Observatory (PAO) found an association of intermediate-scale anisotropies in UHECR arrival directions with the active galactic nuclei (AGNs) and starburst galaxies (SBGs) [19]. A better fit is obtained for the starburst model, disfavoring isotropy at $4.0 \sigma$ statistical significance. Nevertheless, a direct correlation of any UHECR event with an astrophysical source is yet to be found. UHECRs being charged particles are deflected by Galactic and extragalactic magnetic fields, making it difficult to identify their sources. Knowledge of the mass composition at injection is important to understand the nature and properties of these extreme-energy cosmic accelerators.

UHECR events are detected by the PAO in Argentina and the Telescope Array (TA) experiment in the United States from the extensive air showers they induce in the Earth's atmosphere [20, 21]. The atmospheric depth $X_{\max }$ at which the number of secondary particles reaches its maximum can provide insight into the mass composition of the parent UHECR. But the reconstruction of $X_{\max }$ for a UHECR with given energy involves uncertainties due to hadronic interaction models, which are not well known at these extreme energies [2]. The recently measured values of $X_{\max }$ and its fluctuations $\sigma\left(X_{\max }\right)$ by the PAO suggests a progressively heavier composition with increasing energy above $10^{18.3} \mathrm{eV}$ [22]. Thus the long prevalent assumption that UHECRs are pure protons is not viable anymore. Similar conclusions are also drawn from studies of maximum possible cosmogenic photon and neutrino fluxes for a pure proton composition [23].

UHECRs interact with the cosmic microwave background (CMB) and extragalactic background light (EBL), while propagating from their sources to the Earth, producing secondary neutrinos and photons. The main energy loss processes for UHECR protons are Bethe-Heitler pair production and photopion production. Neutrinos are produced from the decays of charged pions, $\pi^{+} \rightarrow \mu^{+}+v_{\mu} \rightarrow \mathrm{e}^{+}+v_{\mathrm{e}}+\bar{v}_{\mu}+v_{\mu}$, and from the neutron beta decay, $n \rightarrow p+\mathrm{e}^{-}+\bar{v}_{e}$. If we consider only the charged pion decay the neutrinos are produced with an initial flavor ratio of $v_{\mathrm{e}}: v_{\mu}: v_{\tau}=1: 2: 0$. The ratio, after propagation over astrophysical distances, is expected to be approximately $1: 1: 1$ when neutrino oscillation is taken into account [24]. The neutron beta decay, as well as, photodisintegration in case the UHECRs are nuclei $(Z>1)$, causes the flavor ratio to deviate from this value. Photodisintegration is the dominant energy loss process for UHECR nuclei, whereby nuclei are irradiated by photons of energies up to $\sim 30 \mathrm{MeV}$ in their rest frame. The flavor ratios of the cosmogenic neutrino fluxes can therefore probe mass composition of UHECRs.

Secondary photons, created by UHECR interactions with the background radiation, can initiate electromagnetic cascades; producing electrons, positrons and gamma photons. The neutrinos, being weakly interacting on the other hand, are only subjected to energy loss due to the adiabatic expansion of the universe; but being chargeless, they point back directly to their sources. These properties make neutrinos an important astrophysical messenger of UHECR acceleration sites. IceCube has detected neutrinos with energies up to a few PeV, and their 9-year data provides a flux upper limit at energies up to $100 \mathrm{EeV} \mathrm{[25].} \mathrm{The} \mathrm{PAO} \mathrm{also} \mathrm{provides} \mathrm{flux} \mathrm{upper} \mathrm{limits} \mathrm{above} 100 \mathrm{PeV}$. The produced flux of neutrinos increases with an increase in proton fraction at injection. For heav- 
ier elements, the resonant interaction of UHECR nuclei with the CMB photons is pushed to higher energies, and for iron $\left({ }^{56} \mathrm{Fe}\right)$ this can go beyond the observed spectrum. For proton-dominated models, the expected neutrino flux peaks at $\sim 10^{18} \mathrm{eV}$. Interactions on the EBL photons can produce a second peak at lower energies, near to $10^{16} \mathrm{eV}$. The actual shape of the spectrum and the energies at which the flux peaks, depend on UHECR source parameters and composition. We have explored the case of light nuclei at injection comprising of hydrogen $\left({ }^{1} \mathrm{H}\right)$ and helium $\left({ }^{4} \mathrm{He}\right)$, using the Monte Carlo simulation tool CRPropa 3 [26]. We vary the abundance fraction at injection to find suitable fits to the UHECR spectrum and calculate the resulting cosmogenic neutrino fluxes. We present results from our investigation to probe the UHECR mass composition at injection by future measurement of neutrino fluxes of individual flavors.

\section{Simulation setup}

We use CRPropa 3, a Monte Carlo simulation code to propagate the UHECRs from the source to Earth [27]. We inject particles from the sources according to a spectrum given by,

$$
\frac{\mathrm{d} N}{\mathrm{~d} E}=A_{0} \sum_{i} K_{i}\left(\frac{E}{E_{0}}\right)^{-\alpha} \begin{cases}1 & \left(E<Z R_{\text {cut }}\right) \\ \exp \left(-\frac{E}{Z R_{\text {cut }}}\right) & \left(E>Z R_{\text {cut }}\right)\end{cases}
$$

where $K_{i}$ is the abundance fraction of the $i-$ th nuclei at injection, $E$ is the energy of the injected particle, $A_{0}$ is an arbitrary normalization flux, $\alpha$ is the spectral index, $Z$ is the charge of the primary cosmic ray and $R_{\text {cut }}=E_{\text {cut }} / Z$ is the cutoff rigidity. We assume the sources inject particles between $E_{\min }=0.1 \mathrm{EeV}$ to $E_{\max }=1000 \mathrm{EeV}$. We consider three cases of injection spectral index $(\alpha)$, viz., 2.2, 2.4, 2.6, and vary the abundance fraction of $\mathrm{H} \& \mathrm{He}$ from 0 to $100 \%$ in steps of $0.1 \%$ restricted by the condition $K_{\mathrm{H}}+K_{\mathrm{He}}=100 \%$. We assume the sources evolve according to a power law in redshift $(1+z)^{m}$, where $z_{\max }$ is varied through $2,3,4$ and $m$ through $0,1,2,3$. The cutoff rigidity $R_{\text {cut }}$ is varied from 50 to $100 \mathrm{EV}$. We add all interaction processes implemented in CRPropa 3, namely, photopion production, Bethe-Heitler interaction, photodisintegration, nuclear decay and energy losses due to expansion of the universe. We consider both CMB and EBL as the background photons for all photohadronic processes. The Dominguéz et al. EBL model is used for the simulations. Neutrinos are also propagated and stored on reaching the observer. Since the energy loss of UHECRs is very small in the galactic and extragalactic magnetic fields, we do not consider them. For propagation of electromagnetic particles, a uniform extragalactic magnetic field of strength $0.1 \mathrm{nG}$ is considered in DINT.

\section{UHECR and cosmogenic neutrino fluxes}

We find the best-fit cases corresponding to each value of $\alpha$ considered in the study, based on a simple $\chi^{2}$ formalism [27]. In Fig. 1, we show the UHECR spectrum on the left and the corresponding cosmogenic neutrino flux on the right. The $\chi^{2}$ analysis was done using the data points in the unshaded energy range only. The required helium to proton fraction $K_{\mathrm{He}} / K_{\mathrm{H}}$ decreases with increase in $\alpha$. All $\alpha=2.6$ cases correspond to pure proton cases and are thus disfavored. However, the $m=0$ cases for $\alpha=2.6$ cases yield better fits than that for $m>0$. On the contrary, 

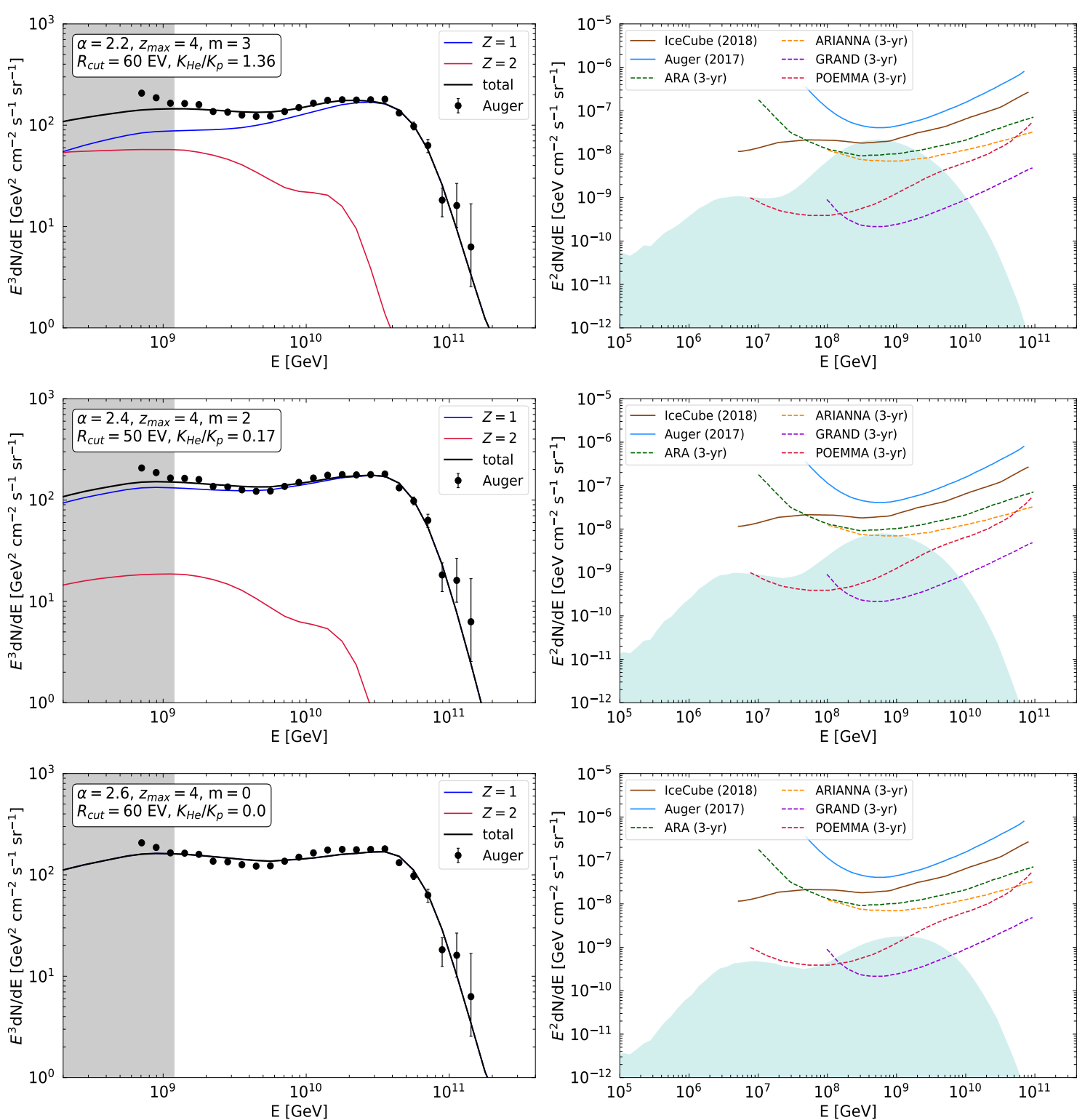

Figure 1: Fits to the PAO UHECR spectra (left) and the corresponding expected cosmogenic neutrino spectra of all flavors (right) for $\alpha=2.2,2.4$, and 2.6 from the top to bottom panels. Also shown (right) are the flux upper limits and sensitivities of the current and future neutrino detectors. Figure adapted from [27].

the fit improves with an increase in $m$ value for $\alpha=2.2$ and 2.4. The $m=3$ cases are disfavored for $\alpha=2.4$, owing to proton domination in the best-fit composition. The best-fit cases are found to produce neutrino spectrum within the flux upper limit imposed by the IceCube and PAO. The sensitivity curves for future neutrino detectors like GRAND [28, 29] and POEMMA [30] indicate that they would be able to constrain the composition models within a few years of observation. See [27] for a detailed analysis.

We have also fitted the PAO spectrum using heavy nuclei at injection $(\mathrm{N}, \mathrm{Si}$ and $\mathrm{Fe}$ in addition to $\mathrm{H}$ and $\mathrm{He}$ ) as shown in Fig. 2. In all cases, the contribution of $\mathrm{Fe}$ in the final fit to the PAO 

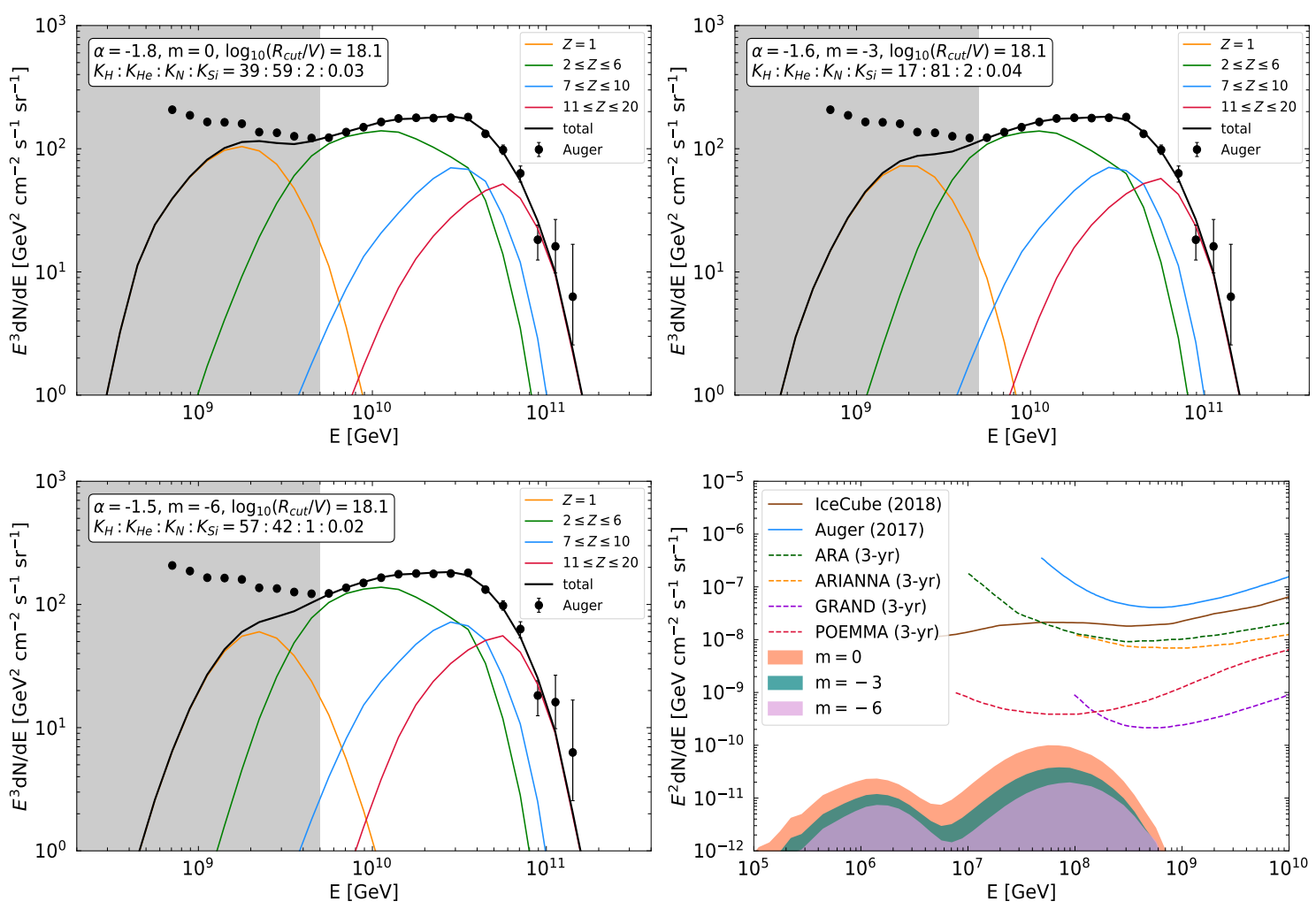

Figure 2: Fits to the PAO UHECR spectra using heavy nuclei mass composition for the parameter values $m=0$ (top left), $m=-3$ (top right) and $m=-6$ (bottom left); as well as the expected neutrino spectra (bottom right) for these fits. Figure adapted from [27].

spectrum is negligible. Furthermore, these fits require very hard injection spectrum $(\alpha<2)$ and negative or no evolution of the UHECR sources $(m \leq 0)$. The resulting neutrino spectra for these cases are also shown in Fig. 2, which are at much lower levels to be detected by any of the current or planned neutrino detectors.

The neutrinos travel distances long enough to undergo flavor conversion from $v_{\alpha}$ to $v_{\beta}$ with a probability given by $P_{\alpha \beta}=\sum_{j=1}^{3}\left|U_{\beta j}\right|^{2} \cdot\left|U_{\alpha j}\right|^{2}$, where $\alpha, \beta=e, \mu, \tau$ and $U$ is the PontecorvoMaki-Nakagawa-Sakata (PMNS) mixing matrix between the neutrino flavor and mass eigenstates. We use the current best-fit values of the mixing angles (for the normal mass hierarchy): $\sin ^{2} \theta_{12}=$ $0.297, \sin ^{2} \theta_{23}=0.425, \sin ^{2} \theta_{13}=0.0215$ and the CP-violating phase $\delta=1.38 \pi$ [31]. The probability matrix is given by

$$
\left(\begin{array}{lll}
P_{e e} & P_{e \mu} & P_{e \tau} \\
P_{\mu e} & P_{\mu \mu} & P_{\mu \tau} \\
P_{\tau e} & P_{\tau \mu} & P_{\tau \tau}
\end{array}\right) \approx\left(\begin{array}{ccc}
0.56 & 0.24 & 0.20 \\
\ldots & 0.38 & 0.38 \\
\ldots & \ldots & 0.42
\end{array}\right)
$$

which is the same for neutrinos and antineutrinos with $P_{\alpha \beta}=P_{\beta \alpha}$. The value of the CP-violating phase $\delta$ can be probed with precise knowledge of the mixing angles and cosmogenic fluxes. The neutrino fluxes of different flavors on the Earth after oscillation is given by

$$
\Phi_{v_{\alpha}+\bar{v}_{\alpha}}=P_{e \alpha}\left(\Phi_{v_{e}}^{0}+\Phi_{\bar{v}_{e}}^{0}\right)+P_{\mu \alpha}\left(\Phi_{v_{\mu}}^{0}+\Phi_{\bar{v}_{\mu}}^{0}\right)+P_{\tau \alpha}\left(\Phi_{v_{\tau}}^{0}+\Phi_{\bar{v}_{\tau}}^{0}\right)
$$



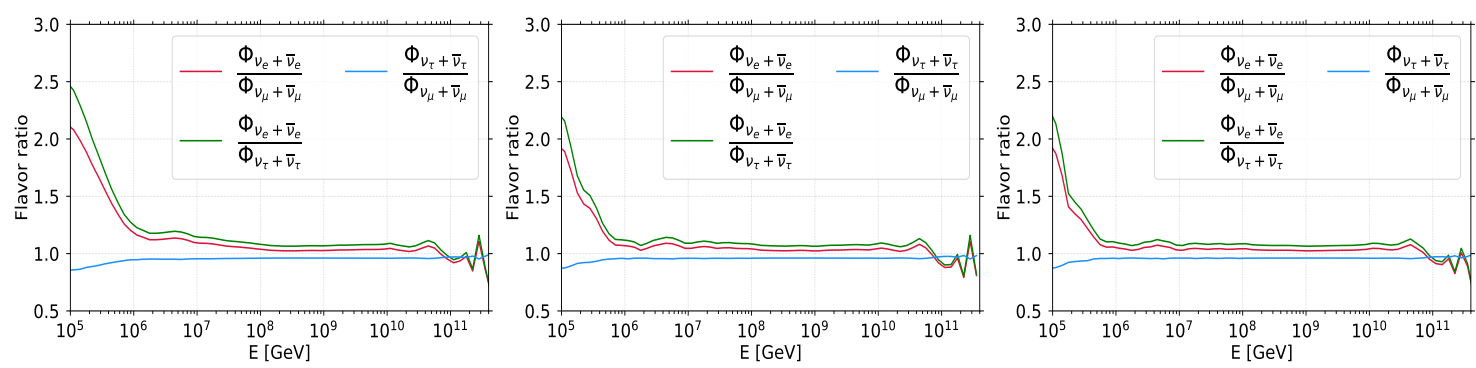

Figure 3: The expected ratio of neutrino fluxes of different flavors, in case of $\mathrm{H}+\mathrm{He}$ composition, for the best fit cases corresponding to $\alpha=2.2,2.4$ and 2.6, respectively from the left to right. Figure adapted from [27].

where $\Phi_{\alpha}^{0}$ are the fluxes generated by the CRPropa code. The neutrino events observed by IceCube are detected from the deep-inelastic neutrino-nucleon scattering. The main signatures to distinguish neutrino events in IceCube are "track-like" events arising from muons produced in charged-current (CC) interactions of $v_{\mu}$ and "shower-like" events generated in neutral-current (NC) interactions of all neutrino flavors, as well as CC interactions for $v_{\mathrm{e}}$ and $v_{\tau}$. In addition to these, high energy $v_{\tau}$ can produce the "double-bang" and other event signatures [32, 33, 34, 35]. The ratios of cosmogenic fluxes of different flavors can be written as

$$
r_{\alpha / \beta}=\frac{\Phi_{v_{\alpha}+\bar{v}_{\alpha}}}{\Phi_{v_{\beta}+\bar{v}_{\beta}}}
$$

For typical $1: 2: 0$ initial flavor ratios, the expected ratio on the Earth is just ratio of the probabilities given as

$$
r_{\alpha / \beta}=\frac{P_{e \alpha}+2 P_{\mu \alpha}}{P_{e \beta}+2 P_{\mu \beta}}
$$

In Fig. 3, the ratios $r_{e / \mu}, r_{\tau / \mu}$ and $r_{e / \tau}$ obtained at the Earth in case of $\mathrm{H}+\mathrm{He}$ composition are shown for $\alpha=2.2,2.4$ and 2.6, from the left to right panels. It can be seen that the ratios are constant below $10^{20} \mathrm{eV}$ up to a certain energy determined by the abundance fraction $K_{\mathrm{He}} / K_{\mathrm{H}}$ at the injection. For higher values of $\alpha$, the $K_{\mathrm{He}} / K_{\mathrm{H}}$ values are lower and the departure occurs at lower values of the neutrino energy. The region with constant ratio has values near to that expected from equation (3.3) for initial pion-decay flavor ratios $1: 2: 0$. A shift from these values at the lower energies is due to neutron beta decays, which is an indicator of the He/p ratio of the UHECR flux at the injection.

\section{Conclusions}

Detection of cosmogenic neutrinos will illuminate the particle acceleration process inside the candidate source classes. However, the UHECR sources can also produce neutrinos at these high energies. So the extrapolated sensitivity of IceCube is truly an upper limit, and a demarcation between astrophysical and cosmogenic neutrinos will be crucial in the future to study their spectrum. Information can be deduced from the observed spectrum, the flavor composition and possible correlation of neutrino events with UHECRs [36, 37], as well as, with candidate source catalogs. The ankle at $E \approx 5 \times 10^{18} \mathrm{eV}$ and the cutoff beyond $E \approx 6 \times 10^{19} \mathrm{eV}$ are the most prominent features 
in the observed spectrum. For light nuclei $(\mathrm{H}+\mathrm{He})$ at injection, the hardening of the spectrum near the ankle can be interpreted as due to a changing composition or $\mathrm{e}^{+} \mathrm{e}^{-}$pair production on cosmic background photons (CMB and EBL). Whereas, for heavier nuclei dominating at the highest energies, the ankle is due to a transition between two or more different population of sources. The cutoff at the highest energies can be interpreted as increased photopion production on the CMB, called GZK phenomenon or due to maximum acceleration energy at the sources.

For heavier elements, only the particles originating from redshifts $z \lesssim 0.5$ are able to reach earth at the highest energies. Also, a negative redshift evolution is seen to be preferred in the latter case to achieve a better fit to the observed UHECR spectrum. The combined effect of these two factors constrains most UHECR to travel distances lesser than the $p \gamma$ interaction length. In addition, the energy loss through photodisintegration dominates over that through photopion production for nuclei $(Z>1)$. Thus the secondary cosmogenic fluxes are reduced due to fewer pion production, beyond the sensitivity of the proposed future detectors. This also implies that the observed spectrum steepens as a result of limited acceleration energy at the sources. Please see [27, 38] for a detailed discussion. On the contrary, GZK cutoff requires primary UHECR energy to be at least comparable to that for photopion production on CMB. Thus, in case of light nuclei at injection, the steepening is due to increased energy loss of primaries and copious production of charged and neutral pions, thereby increasing the cosmogenic neutrino flux. Hence a future detetion of cosmogenic neutrinos as well as the identification of their flavors at these energies can constrain the mass composition and give a plausible explanation of the spectral features. Future detectors like GRAND [28, 29], POEMMA [30] will have much higher sensitivities and may unveil these mysteries.

\section{References}

[1] K. Kotera and A.V. Olinto, Annu. Rev. Astron. Astrophys. 49 (2011) 119 [arXiv: 1101.4256 ]

[2] R. Alves Batista et al., Front. Astron. Space Sci. 6 (2019) 23 [arXiv: 1903.06714 ].

[3] C. D. Dermer and S. Razzaque, Astrophys. J. 724 (2010) 1366 [arXiv: 1004.4249 ].

[4] M. Eichmann, J. P. Rachen, L. Merten, A. van Vliet, J. B. Tjus, JCAP 1802 (2018) 036 [arXiv:1701.06792]

[5] B. Eichmann, JPCS 1181 (2018) 012028 [arXiv: 1812.09473 ]

[6] B. T. Zhang, K. Murase, F. Oikonomou and Z. Li, Phys. Rev. D 96 (2017) 063007 [arXiv:1706.00391]

[7] C. Guépin, K. Kotera, E. Barausse, K. Fang and K. Murase, Astron. Astrophys. 616 (2018) A179 [arXiv:1711.11274]

[8] P. Blasi, R. I. Epstein and A. V. Olinto, Astrophys. J. 533 (2000) L123 [arXiv:astro-ph/9912240]

[9] T. Herpay, S. Razzaque, A. Patkos and P. Meszaros, JCAP 0808 (2008) 025 [arXiv : 0807 . 4914].

[10] S. S. Kimura, K. Murase and P. Mészáros, Astrophys. J. 866 (2018) 51 [arXiv: 1807. 03290].

[11] E. Waxman, Phys. Rev. Lett. 75 (1995) 386 [arXiv: astro-ph/9505082]

[12] M. Vietri, Astrophys. J. 453 (1995) 883 [arXiv: ast ro-ph/9506081]. 
[13] K. Murase, K. Ioka, S. Nagataki and T. Nakamura, Astrophys. J. 651 (2006) L5 [arXiv:astro-ph/0607104]

[14] N. Gupta and B. Zhang, Astropart. Phys. 27 (2007) 386 [arXiv: astro-ph/ 0606744 ].

[15] X. Y. Wang, S. Razzaque, P. Meszaros and Z. G. Dai, Phys. Rev. D 76 (2007) 083009 [arXiv:0705.0027].

[16] R. Budnik, B. Katz, A. MacFadyen and E. Waxman, Astrophys. J. 673 (2008) 928 [arXiv:0705.0041].

[17] C. D. Dermer, S. Razzaque, J. D. Finke and A. Atoyan, New J. Phys. 11 (2009) 065016 [arXiv:0811.1160]

[18] X. Wang and A. Loeb, Phys. Rev. D 95 (2017) 063007 [arXiv: 1611.07616 ]

[19] A. Aab et al. (Pierre Auger Collaboration), Science 357 (2017) 1266 [arXiv: 1709.07321 ]

[20] A. Aab et al. (Pierre Auger Collaboration), JCAP 1508 (2015) 049 [arXiv: 1503.07786 ]

[21] R. U. Abbasi et al., Astrophys. J. 804 (2015) 133 [arXiv: 1407.6145$]$

[22] A. Aab et al. (Pierre Auger Collaboration), journal 1704 (2017) 038 [arXiv: 1612 . 07155]

[23] A. D. Supanitsky, Phys. Rev. D 94 (2016) 063002 [arXiv: 1607 . 00290]

[24] S. Pakvasa, W. Rodejohann and T. J. Weiler, JHEP 0802 (2008) 005 [arXiv: 0711.4517 ].

[25] M. G. Aartsen et al. (IceCube Collaboration), Phys. Rev. D 98 (2018) 062003 [arXiv:1807.01820]

[26] R. A. Batista et al., JCAP 1605 (2016) 038 [arXiv: 1603.07142 ]

[27] S. Das, S. Razzaque and N. Gupta, Phys. Rev. D 99 (2019) 083015 [arXiv: 1809.05321 ]

[28] O. Martineau-Huynh et al., EPJ Web Conf. 135 (2017) 02001 [arXiv: 1702 . 01395 ]

[29] K. Møller, P. B. Denton and I. Tamborra, JCAP 1905 (2019) 047 [arXiv: 1809.04866 ]

[30] A. V. Olinto et al., PoS ICRC2017 (2017) 542

[31] F. Capozzi et al., Phys. Rev. D 95 (2017) 096014 [arXiv: 1703.04471 ]

[32] J. G. Learned and S. Pakvasa, Astropart. Phys. 3 (1995) 267 [arXiv: hep-ph/ 9405296 ].

[33] J. F. Beacom et al., Phys. Rev. D 68 (2003) 093005 [arXiv: hep-ph/0307025]

[34] T. DeYoung, S. Razzaque and D. F. Cowen, Astropart. Phys. 27 (2007) 238 [arXiv:astro-ph/0608486]

[35] M. G. Aartsen et al. (IceCube Collaboration), Adv. Space. Res. 62 (2018) 2902 [arXiv: 1701.03731]

[36] R. Moharana and S. Razzaque, JCAP 1508 (2015) 014 [arXiv: 1501 . 05158].

[37] M. G. Aartsen et al. [IceCube and Pierre Auger and Telescope Array Collaborations], JCAP 1601 (2016) 037 [arXiv: 1511.09408].

[38] R. A. Batista, R. M. de Almeida, B. Lago and K. Kotera, JCAP 1901 (2019) 002 [arXiv:1806.10879] 